\title{
MOBS, TREES, AND FIXED POINTS
}

L. E. WARD, JR.

1. Introduction. In an earlier paper [5] the author characterized dendrites and trees in terms of partially ordered spaces, and this characterization has been employed $[2 ; 6]$ to give an order-theoretic proof of the well-known theorem that every tree has the fixed point property. In the present note three more characterizations of trees are given; in $\$ 2$ they are shown to be precisely a special class of mobs and in $\$ 3$ order-theoretic and purely topological formulations are given. A notion of generalized tree is introduced and a fixed point theorem is proved. This theorem contains the fixed point theorem for trees and the recent result of $\mathrm{K}$. Borsuk [1] that an arcwise connected acyclic curve has the fixed point property.

2. Mobs and partially ordered spaces. A partially ordered topological space (abbreviated POTS) is a space $X$ together with a partial order $\leqq$ defined on $X$ such that $\leqq$ is semicontinuous in the sense that

$$
L(x)=\{a: a \leqq x\} \text { and } M(x)=\{a: x \leqq a\}
$$

are closed sets for each $x \in X$. The partial order is continuous if it has a closed graph in $X \times X$. These spaces have been studied in [4]. A partially ordered set is order-dense if there exists $z$ with $x<z<y$ whenever $x<y$. A zero of a partially ordered set is an element which precedes all other elements of the set. We shall frequently write

$$
[x, y]=M(x) \cap L(y) \text {, }
$$

and

$$
(x, y)=[x, y]-x \cup y \text {. }
$$

An important class of POTS's is provided by the so-called cutpoint ordering of locally connected continua. (In this paper a continuum is a compact connected Hausdorff space.) If $K$ is a locally connected continuum, we choose $e \in K$ and define $x \leqq y$ if and only if $x=e$ or $x=y$ or $x$ separates $e$ and $y$ in $K$. It was proved in [4] that $K$, partially ordered in this manner, is a POTS.

A tree is a special type of locally connected continuum. It is a continuum in which every pair of points is separated by some third point. The following result was proved in [5].

Presented to the Society, November 17, 1956; received by the editors November 26, 1956. 
TheOREM 1. Let $X$ be a compact Hausdorff space. A necessary and sufficient condition that $X$ be a tree is that $X$ admit a partial order $\leqq$ such that

(i) $\leqq i s ~ s e m i c o n t i n u o u s$,

(ii) $\leqq$ is order-dense,

(iii) if $x \in X$ and $y \in X$, then $L(x) \cap L(y)$ is a nonempty chain,

(iv) if $x \in X$, then $M(x)-x$ is an open set.

In addition, we note

THEOREM 2. If $X$ is a tree then $\leqq$ is continuous.

Proof. It suffices to show that if $x y$ then there exist open sets $U$ and $V$, with $x \in U$ and $y \in V$, such that $a \neq b$ whenever $a \in U$ and $b \in V$. Since $X$ is order-dense we may choose $t \in X$ such that $t<x$ and $t \neq y$. If $U=M(t)-t$ and $V=X-M(t)$ then $U$ and $V$ are open sets with the desired properties.

It is easy to see that this ordering of a tree is always the cutpoint ordering.

A $m o b$ as defined by Wallace and others is an associative Hausdorff semigroup with continuous multiplication. There is an extensive bibliography on mobs in [3]. The mob $S$ is monotone if multiplication is a monotone function, i.e., if the set of pairs $(a, b)$ such that $a b=x$ is connected in $S \times S$ for all $x \in S$. Define $x \leqq y$ in $S$ if and only if $x y=x$. This relation is transitive but is not, in general, a partial order and may even be vacuous.

THEOREM 3. If $S$ is an idempotent commutative mob, then $S$ is a POTS. If, in addition, $L(x)$ is compact for some $x \in S$, then $S$ is a POTS with zero.

PROof. That $\leqq$ is reflexive, anti-symmetric, and transitive (and hence a partial order) follows from the idempotence, commutativity, and associativity, respectively, of $S$. To show that $M(x)$ is closed for all $x \in S$, suppose $y \in S-M(x)$ and let $V=S-x$. Since multiplication is continuous there exists a neighborhood $U$ of $y$ such that $U x \subset V$. If there exists $t \in U \cap M(x)$ then $t x=x \in V$ which is a contradiction. To show that $L(x)$ is closed, suppose $y \in S-L(x)$ and $V$ is an open set such that

$$
x y \in V \subset \bar{V} \subset S-y .
$$

Again by continuity a neighborhood $U$ of $y$ may be chosen such that $U$ and $V$ are disjoint and $U x \subset V$. If there exists $t \in U \cap L(x)$ then $t x=t \in U \cap V$ which is a contradiction. Thus $S$ is a POTS. The exist- 
ence of a zero in case some $L(x)$ is compact is an immediate consequence of Theorem 1 of [4].

For the remainder of this section it is understood that

$$
\begin{aligned}
& m^{-1}(a)=\{(x, y): x y=a\}, \\
& m_{b}^{-1}(a)=\{x: x b=a\} .
\end{aligned}
$$

THEOREM 4. If $S$ is an idempotent monotone mob then $S$ is orderdense.

Proof. If $S$ is not order-dense then there exist elements $x$ and $y$ of $S$ such that $x<y$ and $(x, y)$ is empty. Therefore

$$
m_{y}^{-1}(x)=M(x)-M(y) .
$$

Moreover, the transitivity of $\leqq$ implies that $M(y) \subset M(x)$ and consequently

$$
M(x)=m_{y}^{-1}(x) \cup M(y),
$$

which is a decomposition of $M(x)$ into disjoint nonempty closed sets. On the other hand, $M(x)=\pi m^{-1}(x)$, where $\pi: X \times X \rightarrow X$ is the projection; since $m$ is monotone, $M(x)$ is connected. This is a contradiction.

Theorem 5. A necessary and sufficient condition that $X$ be a tree is that $X$ be a compact idempotent commutative monotone mob such that if $a x=a$ and $b x=b$, then $a b=a$ or $a b=b$.

Proof. Sufficiency. According to Theorems 1, 3, and 4 it is suffcient to show that, if $X$ is a mob of the desired type, then $L(x) \cap L(y)$ is always a nonempty chain and $M(x)-x$ is always open. That $L(x)$ is a chain follows from the condition: $a x=a$ and $b x=b$ implies $a b=a$ or $a b=b$; since $X$ has a zero, $L(x) \cap L(y)$ is nonempty. Suppose $y \in M(x)-M(x)^{0}$, and let $V$ be a neighborhood of $y=y^{2}$. By continuity there is a neighborhood $U$ of $y$ such that $U y \subset V$. Since $U$ meets $X-M(x)$ it follows that $U y$ meets $L(x)$ and hence $y \in \bar{L}(x)$ $=L(x)$. Therefore $x=y$ so that $M(x)^{0}=M(x)-x$.

Necessity. If $X$ is a tree endowed with the partial order of Theorem 1 , then the operation

$$
x y=\sup L(x) \cap L(y)
$$

is obviously commutative and idempotent; further, the fact that $L(x)$ is a chain implies that whenever $a x=a$ and $b x=b$, then $a b=a$ or $a b=b$. To see that multiplication is continuous, consider the case where $x$ and $y$ are not comparable. (The case $x \leqq y$ follows in a similar 
manner.) Let $U$ be a neighborhood of $x y$ and let $V_{x}=M(t)-t, V_{y}$ $=M(s)-s$ where $t$ and $s$ are chosen so that $x y<t<x, x y<s<y$. The sets $V_{x}$ and $V_{y}$ are neighborhoods of $x$ and $y$ respectively and

$$
V_{x} V_{y}=x y \in U .
$$

Finally, to show that multiplication is monotone we note that

$$
m^{-1}(z)=\pi m^{-1}(z) \times \pi m^{-1}(z)=M(z) \times M(z) .
$$

Since $M(z)$ is connected, so is $m^{-1}(z)$.

The previously mentioned fixed point theorem for trees can now be stated in the language of mobs. However, in $\$ 4$ a more general result will be obtained.

3. Generalized trees and hereditary unicoherence. Modifications of conditions (i) and (iv) of Theorem 1 are introduced.

$\left(i^{\prime}\right) \leqq$ is continuous,

(iv') if $Y$ is a closed and connected subset of $X$, then $Y$ contains a zero.

A compact Hausdorff space $X$ is said to be a generalized tree if and only if $X$ admits a partial order satisfying conditions (i'), (ii), (iii), and $\left(\mathrm{iv}^{\prime}\right)$. From Theorems 1 and 2, and the fact that the property of being a tree is hereditary with respect to subcontinua, we have

Theorem 6. A tree is a generalized tree.

It is easy to construct examples of generalized trees which are not locally connected and hence are not trees, so that the term "generalized" tree is an appropriate one. For example, in the plane let

$$
\begin{aligned}
A_{-1} & =\{(x, 0): 0 \leqq x \leqq 1\}, \\
A_{0} & =\{(0, y): 0 \leqq y \leqq 1\}, \\
A_{n} & =\left\{\left(\frac{1}{n}, y\right): 0 \leqq y \leqq 1\right\}, \quad n=1,2, \cdots
\end{aligned}
$$

and define

$$
X=\bigcup_{n=-1}^{\infty}\left\{A_{n}\right\}
$$

Define $\left(x_{1}, y_{1}\right) \leqq\left(x_{2}, y_{2}\right)$ if and only if $x_{1} \leqq x_{2}$ and $y_{1}=0$, or $x_{1}=x_{2}$ and $y_{1} \leqq y_{2}$. It may be readily verified that $X$, together with this partial order, is a generalized tree. However, $X$ is not locally connected.

TheOREM 7. A generalized tree is a hereditarily unicoherent continuum. Conversely, a hereditarily unicoherent continuum which admits 
a partial order with zero satisfying ( $\left.\mathrm{i}^{\prime}\right)$ and (ii) is a generalized tree.

Proor. The order-denseness of the partial order ensures that, if $X$ is a generalized tree, then $X$ is connected and hence is a continuum. To demonstrate that $X$ is hereditarily unicoherent it will first be shown that if $A$ is a subcontinuum of $X$ and if $a$ and $b$ are elements of $A$ such that $a<b$ then $[a, b] \subset A$. For if not then $a$ and $b$ may be so chosen that $(a, b) \cap A$ is empty. Let $a<p<b$ and let $U$ be an open set such that $L(a) \subset U$ and $\bar{U} \cap M(p)$ is empty. If $B$ is the component of $A-U$ which contains $b$, then according to (iv') $B$ must have a zero, but, since $B \cap L(b)=b$, that zero must be $b$ itself and hence $B \subset M(b)$. Therefore $B \cap \bar{U}$ is empty, which contradicts a well-known theorem that $\bar{U}$ must contain a limit point of each component of $A-\bar{U}$. Consequently $[a, b] \subset A$. Suppose now that $A$ and $B$ are subcontinua of $X$ and that $x$ and $y$ are elements of $A \cap B$. If $z=\sup L(x)$ $\cap L(y)$, it follows that

$$
Z=[z, x] \cup[z, y] \subset A \cap B .
$$

Since $Z$ is connected, so is $A \cap B$ and therefore $X$ is hereditarily unicoherent.

To prove the converse, assume that $X$ is a hereditarily unicoherent continuum admitting a partial order which is continuous, orderdense, and has a zero. If $x \in X$ and if $L(x)$ contains noncomparable points $a$ and $b$, then the continua $L(a) \cup[a, x]$ and $L(b) \cup[b, x]$ have a nonconnected intersection. Thus the hereditary unicoherence of $X$ implies that $L(x)$ is a chain. If $Y$ is a subcontinuum of $X$ and if $Y$ contains two distinct minimal elements, $t_{1}$ and $t_{2}$, then the continua $L\left(t_{1}\right) \cup L\left(t_{2}\right)$ and $Y$ have a nonconnected intersection. Therefore $Y$ has a zero, and the theorem is proved.

We conclude this section with two more characterizations of trees.

TheOREM 8. Let $X$ be a locally connected continuum. A necessary and sufficient condition that $X$ be a tree is that the cutpoint ordering be orderdense.

Proof. The necessity follows at once from [5]. To prove the sufficiency we must show that if $x$ and $y$ are distinct elements of $X$ then there exists $p \in X$ separating $x$ and $y$. If $x<y$ in the cutpoint ordering then $x=e$ or

$$
\begin{gathered}
X-x=A_{x} \cup B_{x}, \quad A_{x} \mid B_{x}, \\
e \in A_{x}, \quad y \in B_{x},
\end{gathered}
$$

where $A_{x}$ is a component of $X-x$. Let $x<p<y$. If $x=e$ then $p$ sepa- 
rates $x$ and $y$ by the definition of the cutpoint ordering. If $x \neq e$ then

$$
\begin{gathered}
X-p=A_{p} \cup B_{p}, \quad A_{p} \mid B_{p}, \\
e \in A_{p}, \quad y \in B_{p},
\end{gathered}
$$

and $p \in B_{x}$. Since $A_{x}$ is connected it follows that $A_{x} \subset A_{p}$ so that $p$ separates $x$ and $y$. If $x$ and $y$ are not comparable, let $z=\sup L(x) \cap L(y)$ and choose $p$ such that $z<p<y$. As in the case just considered the separation (1) occurs with $z \in A_{p}$. Since $B_{p} \cup_{p}=M(p)$ and $p \nless x$, it follows that $p$ separates $x$ and $y$.

THEOREM 9. A necessary and sufficient condition that $X$ be a tree is that $X$ be a hereditarily unicoherent locally connected continuum.

Proof. Necessity. By Theorem 7 any generalized tree, and hence any tree, is hereditarily unicoherent. By Lemma 4 of [5], a tree is a locally connected continuum.

Sufficiency. Fix $e \in X$; it is clear that hereditary unicoherence implies that for each $x \in X$ there is a unique irreducible continuum $L(x)$ joining $e$ and $x$. Let $x \leqq y$ mean that $L(x) \subset L(y)$. To complete the proof it is sufficient to show that $\leqq$ is the cutpoint ordering. Suppose

$$
X-p=A_{p} \cup B_{p}, \quad A_{p} \mid B_{p}, \quad e \in A_{p},
$$

where $A_{p}$ is connected. Clearly, $L(p) \subset L(x)$ if and only if $x \in B_{p}$, i.e., if $p$ separates $e$ and $x$.

4. A fixed point theorem. The following theorem is a slight generalization of the recent result of $\mathrm{K}$. Borsuk mentioned in $\$ 1$. They coincide for generalized trees in which chains are assumed to be separable. The methods of proof are substantially different.

TheOREM 10. If $X$ is a generalized tree and $f(X) \subset X$ is continuous, then $f(x)=x$ for some $x \in X$.

Proof. Since $X$ has a zero, the set

$$
P=\{x: x \leqq f(x)\}
$$

is nonempty. Let $C$ be a maximal chain of $P$ and $x=\sup C$; we first show that $x \in P$ and hence that $P$ contains a maximal element. If $x \in X-P$ then

$$
y=\sup L(x) \cap L(f(x))<x
$$

and there exists in $C$ an increasing net $x_{\alpha}$ such that $y<x_{\alpha}<x$ and $\lim x_{\alpha}=x$. Since $f\left(x_{\alpha}\right) \in M\left(x_{\alpha}\right)$ for each $\alpha$ and since $\lim f\left(x_{\alpha}\right)=f(x)$ it follows that

$$
f(x) \in \bigcap_{\alpha}\left\{M\left(x_{\alpha}\right)\right\}=K .
$$


The set $K$, being the intersection of a nested collection of continua, is a continuum, and therefore $K$ has a zero, $k$. Thus $k$ is a proper predecessor of both $x$ and $f(x)$ and it follows that, for some $\alpha, k<x_{\alpha}$. But this implies that $M\left(x_{\alpha}\right)$ fails to contain $K$, a contradiction. Thus $x \leqq f(x)$ and $x$ is maximal with respect to this property. If $x<f(x)$ let $x<z<f(x)$, and note that, by the maximality of $x$ in $P$,

$$
f([x, z]) \cap M(z)=f(x) .
$$

Consequently the two continua $[z, f(x)]$ and

$$
f([x, z]) \cup L(f(z)) \cup L(z)
$$

meet in the disconnected set $z \cup f(x)$, which contradicts the hereditary unicoherence of $X$. Therefore $x=f(x)$.

\section{REFERENCES}

1. K. Borsuk, $A$ theorem on fixed points, Bull. Acad. Polon. Sci. Cl III vol. 2 (1954) pp. 17-20.

2. C. E. Capel and W. L. Strother, A new proof of a fixed point theorem of Wallace, Bull. Amer. Math. Soc. Abstract 62-1-111.

3. A. D. Wallace, The structure of topological semigroups, Bull. Amer. Math. Soc. vol. 61 (1955) pp. 95-112.

4. L. E. Ward, Jr., Partially ordered topological spaces, Proc. Amer. Math. Soc. vol. 5 (1954) pp. 144-161.

5. - A note on dendrites and trees, Proc. Amer. Math. Soc. vol. 5 (1954) pp. 992-994.

6. - Fixed point theorems for special types of continua, Bull. Amer. Math. Soc. Abstract 61-3-439.

U. S. Naval Ordnance Test Station, China lake, California 